\title{
Islanding detection in micro-grids using sum of voltage and current wavelet coefficients energy
}

\author{
Hossein Haroonabadi \\ Dep. of Electrical Engineering, Islamshahr Branch, Islamic Azad University, Iran
}

Email address:

haroonabadi@iiau.ac.ir

To cite this article:

Hossein Haroonabadi. Islanding Detection in Micro-Grids Using Sum of Voltage and Current Wavelet Coefficients Energy. International Journal of Energy and Power Engineering. Vol. 3, No. 5, 2014, pp. 228-236. doi: 10.11648/j.jjepe.20140305.12

\begin{abstract}
This paper presents wavelet based islanding detection in distributed generation (DG) interfaced to the microgrid. Also a new fast method is developed for islanding detection based on measuring the utility currents and voltages signals processed by discrete wavelet transform. These currents and voltages signals are measured before the main circuit breaker of microgrid network and their features extracted by discrete wavelet transform. These features are sum of wavelet coefficients energy and are used for distinguishing the islanding conditions from non-islanding ones. Because of changing in measuring point of currents and voltages signals from point of common coupling (PCC) in traditional methods to before the main circuit breaker in proposed method, this new method detects the islanding conditions faster than the other methods. The proposed method has been examined under various scenarios; including mains supply faults, various one, two, or three phases' grid faults, and changes of rate of produced energy on IEEE 1547 anti-islanding test system. The numerical studies show the feasibility and applicability of the proposed method with satisfactory results.
\end{abstract}

Keywords: Islanding Detection, Discrete Wavelet Transform, Distributed Generation (DG), Non detection zone (NDZ)

\section{Introduction}

Increased penetration of distributed generation (DG) in power grids is evident to meet the increasing load demand and promote renewable energy sources. The increased DG penetration increases the distribution system complexity and raises several concerns. Among the most important concerns is islanding. Islanding is a condition in which a portion of the distribution system is comprised of DG, and local loads remains energized while they are unintentionally isolated from the rest of the system. The isolation could be a result of fault occurrence on the main distribution feeder. The re-closer will reconnect the isolated part of the system after a certain time interval (set by the system operator). This action is unfavorable since it may cause damage to the distribution system. Consequently, islanding detection (anti-islanding) becomes an important DG protection requirement.

The islanding phenomenon usually occurs when the output power of the DGs and the load power are balanced, i.e., the load power is entirely supplied by DGs. At this time, if the utility is interrupted or failed, the disturbances of voltage and frequency of the DGs cannot be detected with the standard of UL1741 or IEEE1547 [1, 2].
Islanding detection methods can be divided into two categories: one is the passive islanding detection methods [3]; the other is the active islanding detection methods $[4,5]$. In the passive islanding detection methods, the over/under voltage protection and over/under frequency protection are the basic passive islanding detection method for the DG to detect an islanding condition by monitoring parameters in point of common coupling (PCC) and then cause the DG to shut down when there is sufficient transition from normal specified conditions [3]. The flaw of these passive islanding detection methods are that when the power of DGs and the load are balanced there will exist a large NDZ making the passive islanding detection method fail and may not be reliable in high DG penetration cases. Therefore, the active islanding detection methods have become more important in recent years. Active frequency drift method is by adding dead time to the output current of inverter and results in PCC voltage and current distortion. Thus, when the utility is interrupted or failed, the frequency can drift to shift out of the NDZ [4]. For overcoming this, a new fast method is developed for islanding detection based on measuring the utility currents and voltages signals processed by discrete wavelet transform. These currents and voltages signals are measured before the main circuit breaker of microgrid 
network and their features extracted by discrete wavelet transform.

One of the most important challenges in the near future is the complete integration of the distributed generators (DGs) in electric power systems, especially at distribution level (i.e. in medium and low voltage networks). In fact, such integration would allow the best use of the renewable sources (RS) available on the territory and otherwise not exploitable. This implies a complete rethinking of the management and control of electricity networks, which have to move from passive systems to new active "smart grids" [6]. The existing passive systems are characterized by unidirectional energy flows and a limited amount of intelligent and automation functions. On the contrary, in the smart grids concept, energy flows are bidirectional and smart metering technologies and capabilities are needed, also with a two-way communications network and a number of other intelligent field devices, providing for monitoring, automation, protection and control actions [7]. In this method, measuring the utility currents and voltages signals are sent by two-way communications network smart grids.

Islanding results in several safety and power-quality (PQ) issues, including abnormal variations in frequency and voltage in the power island, possibility of creating an ungrounded system depending on the transformer connections, and potential safety hazards for repair crews from unidentified islands. Most interconnection regulations, which are usually guided by the IEEE standards 1547-2003 [8], recommend immediate disconnection of the DG, upon the formation of an island. This is achieved through anti-islanding protection, which is a subject that has been extensively studied [9]-[18].

Wavelet-transform-based techniques have been previously used for islanding detection in [15]-[17]. In [15] and [16], the absolute value of certain wavelet coefficient (of voltage or frequency signal) is compared against a threshold value; and if the relevant wavelet coefficient remains above this preset threshold for a period longer than a certain time threshold, an islanding condition is declared. These system specific threshold values are determined through trials and/or based on the experience of utility engineers [16]. A hybrid islanding detection algorithm based on wavelet transform, which is specifically for single-phase photovoltaic (PV) DG systems is discussed in [17]. An intelligence-based method is investigated in [18], which uses the decision-tree (DT) classifier, but with some complex set of features, including, total harmonic distortion of current/voltage, gradient of the product of voltage and power factor, etc. for classification.

currents and voltages signals that are measured and their features extracted by discrete wavelet transform is explained in [19] and changing in measuring point of currents and voltages signals from point of common coupling in traditional methods to before the main circuit breaker is explained in [20]. In this article these two methods are mixed together.

For detecting islanding in microgrids, this paper presents an on-line measurement method based on wavelet transform for current and voltage analysis. The remaining paper is organized as follows: Section 2 describes the continuous and discrete wavelet transform. Section 3 addresses proposing islanding detection method. The simulation results and operation of the islanding detection scheme are depicted in Section 4. Lastly, the conclusion is presented in Section 5.

\section{The Wavelet Transform}

Wavelet transform is a kind of time-frequency analysis, which has the characteristic of multi-resolution and can symbolize the signal's characteristic in time-frequency field [21]. The shape of time-frequency window is variable while the size is invariable, namely time window and frequency window both are variable. It has higher frequency resolution and lower time resolution in low frequency segment while higher time resolution and lower frequency resolution in high frequency segment. It's quite fit for detecting voltage signal carries non-stationary signal and showing the components.

\subsection{Continuous Wavelet Transform (CWT)}

Wavelet provides time-scale information of a signal, enabling the extraction of features that vary in time. This property makes wavelets an ideal tool for analyzing signals of a transient or non-stationary nature. The continuous wavelet transform of $f(t)$ is a time-scale method of signal processing that can be defined as the sum over all time of the signal multiplied by scaled, shifted versions of the wavelet function $\psi(t)$. Mathematically,

$$
W_{f}(a, b)=|a|^{-\frac{1}{2}} \int_{-\infty}^{+\infty} f(t) \psi\left(\frac{t-b}{a}\right) d t=\left\langle f, \psi_{a, b}\right\rangle
$$

Where $f(t)$ is the original signal, $\psi(t)$ is the mother wavelet and is defined by:

$$
\psi_{a, b}(t)=|a|^{-\frac{1}{2}} \psi\left(\frac{t-b}{a}\right) \quad a, b \in R, a \neq 0
$$

The parameter a is the scale parameter of the wavelet, the parameter $\mathrm{b}$ is a translation parameter of the wavelet and $W_{f}(a, b)$ is the wavelet transform. Unlike the discrete wavelet transform, any scale a can be chosen up to a maximum. The CWT is also continuous in terms of the shift b during computation; the analyzing wavelet is shifted smoothly over the full domain of the analyzed function. Instead of producing a time frequency map, a time-scale map is produced where the scale represents a frequency range.

In (2), take $a=2 j, b \subset R$, that is only doing binary discrete to scale parameter $\mathrm{a}$, and translation parameter $\mathrm{b}$ still maintains continuous change, we call this kind of wavelet as binary wavelet.

Because binary wavelet transform maintains continuous change of variable t, it does not damage the signal's translation invariant character in $\mathrm{x}$ domain, and then binary wavelet transform is comprehensively applied in pattern recognition and signal's singularity detection. We can see 
from translation invariant character that: signal's sharp change point is one by one corresponding to its modulus maxima of wavelet transform. Due to signal's singular point contains important information, the modulus maxima of wavelet transform can depict the singular point and singularity of non-stationary signal, and then we can use it to detect islanding.

Actually, wavelet transform is to decompose signal layer by layer according to different frequency band, and there will be signal of different frequency band in different scale after decomposing. Generally, the frequency of noise signal is comparative high, so it will exist only in lower scale of wavelet transform; but the frequency content of discontinuous signal is abundant, so there will be peak value in a certain scale range of its wavelet transform. When applying wavelet transform to detect non-stationary signal, the choice of scale parameter should be apt, and it is related to frequency component in non-stationary signal and the range of sampling frequency. In practice, the distribution of modulus maxima of wavelet transform is different in different scale. A modulus maximum exists in one scale, it will unnecessarily appear in another scale. So we can consider the modulus maxima of wavelet transform in several scales to decide the position of singular point.

\subsection{Discrete Wavelet Transform (DWT)}

The discrete wavelet transform (DWT) is a signal processing tool for the detection of the islanding condition when the time localization is required [22]. The DWT of a discrete function $\mathrm{x}(\mathrm{k})$ can be defined as:

$$
\psi_{a, b}(t)=|a|^{-\frac{1}{2}} \psi\left(\frac{t-b}{a}\right) \quad a, b \in R, a \neq 0
$$

where $\mathrm{m}$ and $\mathrm{n}$ are positive integers used to define scaling and translating factors employed to the selected mother wavelet $\psi$. Due to the possible values of $\mathrm{m}$, the frequency bands which can be measured by applying the DWT are logarithmic. It is important that the response time of the anti-islanding detection algorithm will be shorter if a lower decomposition level is selected. As a consequence the 4th decomposition level has been selected. Other parameters, such as the employed mother wavelet and the number of filter coefficients have been adjusted by simulation.

\section{Proposing Islanding Detection Method}

When a fault happens in power network, the fault should be clear and the fault location should be disconnected from power system by circuit breaker. The time for fault clearing is the sum of relay operation time and interrupting fault current time by circuit breaker. Even in instantaneous operation mode of relay, 0.5 to 1 cycle is needed to send the open signal to the circuit breaker. Circuit breaker operation time depends on number of its operation and its type, in range of 3 to 5 cycle. In this paper the utility current and voltage signals are measured at DGs side of utility circuit breaker. Therefore, islanding detection time isn't affected by circuit breaker operation time and it takes time less than the local methods to detect the islanding condition by eliminating the circuit breaker operation time. The proposed measuring point is shown in Fig. 1.

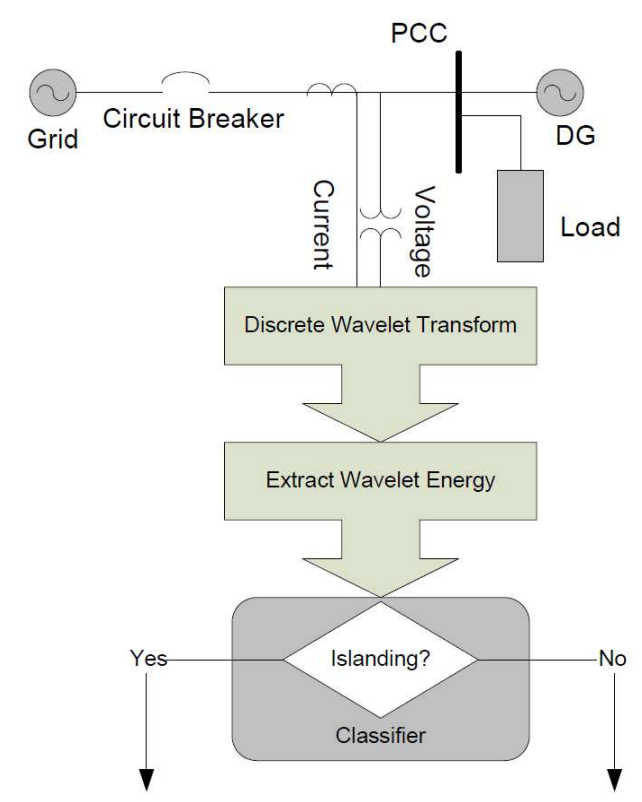

Figure 1. Location of current and voltage measuring point in the proposed Basic model with the transient-based islanding detection technique

The islanding conditions detection in local method start after disconnection or opening of circuit breaker. The system parameters such as voltage, current and frequency are measured in local method and islanding condition is detected when these variations are sensed after the islanding happened. Islanding conditions can be detected before opening of utility circuit breaker, by movement the measuring point from PCC to after the utility circuit breaker. For the islanding detection in the proposed method the wavelet transform used in this paper. The islanding detection time is effectively decreased in proposed method.

In this paper NDZ is eliminated because the conditions which create the islanding are identified by wavelet transform before the circuit breaker opens, while NDZ is created due to difference between active and reactive power when islanding is occurred in local method. No power quality problem exists in the proposed method because there is no disturbance injection. Therefore, this method doesn't have the problems which exist in passive and active method [19].

The output of wavelet transform in this method is the feature which is selected by wavelet process to detect the islanding condition. In this paper, Daubechies wavelet is applied to extract the required feature for islanding detection. Maximum value of DWT forth level is the feature that is selected in this paper. The coefficient of this level is severely different between islanding condition and non islanding ones and can be used to distinguish these conditions accurately.

The basic model of the passive islanding detection method proposed in this paper is illustrated in Fig. 1. Transient 
waveforms of the currents and voltages in a power network contain unique signatures that reveal the cause of the corresponding transient event. The proposing islanding detection method is based on the hypothesis that the transients generated during the islanding event contain such a signature and a classifier can be developed to distinguish islanding events from the other disturbances.

However, the event-specific characteristics embedded in the transient waveforms are not directly distinguishable. Therefore, they need to be pre-processed to extract features that assist fast classification response. Wavelet transformation is thus used for this purpose. Reference [23] by the authors of this paper examines the use of different pattern-recognition techniques for classifying islanding and non-islanding events using transient signals. This paper concludes that DT performs better than probabilistic neural networks or support vector machines. Based on the conclusions arrived in [23], DT was used as the classification technique in this paper [20].

Two kinds of classifier are used and compared to each others, Decision-tree and Artificial Neural Networks Classifier.

\subsection{Artificial Neural Networks Classifier}

Pattern recognition involves different mathematical approaches to classify data (patterns) based either on a priori knowledge or on statistical information extracted from the patterns.

Artificial neural networks (ANNs) have been used in many potential applications in power systems operation and control. Load forecasting, fault diagnosis/fault location, economic dispatch, transient stability and harmonics analysis are some of the application in which ANN was adopted as a classifier [24]. ANNs are often used as classifiers since they have the capability of learning complex mapping, linear or nonlinear from the input space to the output space [25]. The architecture and the training algorithm of the feed forward artificial neural network are used in this article.

A training algorithm is defined as a procedure of updating the weights and biases of a network so the network will be able to perform the particular design task. The training algorithm is divided into two main categorizes: supervised learning, and unsupervised learning. ANN is classified under supervised learning.

\subsection{Decision-Tree Classifier}

DT is a logical model constructed based on the training data, and represented as a binary tree. The DT starts with the "Root," which contains whole training dataset. Each "Internal Node" tests an attribute and each "Arc" corresponds to an attribute value. "Terminal Node" represents the predicted class [26]-[28]. Fig. 2 shows a sample structure of a DT of a two-class problem. Classification and regression trees (CART) [27]-[30] is a nonparametric technique that produces either classification or regression trees, depending on whether the dependent variable is categorical or numeric, respectively. The CART algorithm generates DTs based on a splitting rule. The basic idea of the splitting rule is to choose a split among all possible splits at each node so that the resulting child nodes are the "purest." The splitting rule is processed in three steps as follows:

1) Find the best split of each predictor variable.

2) Find the best split of node.

3) Assign the class.

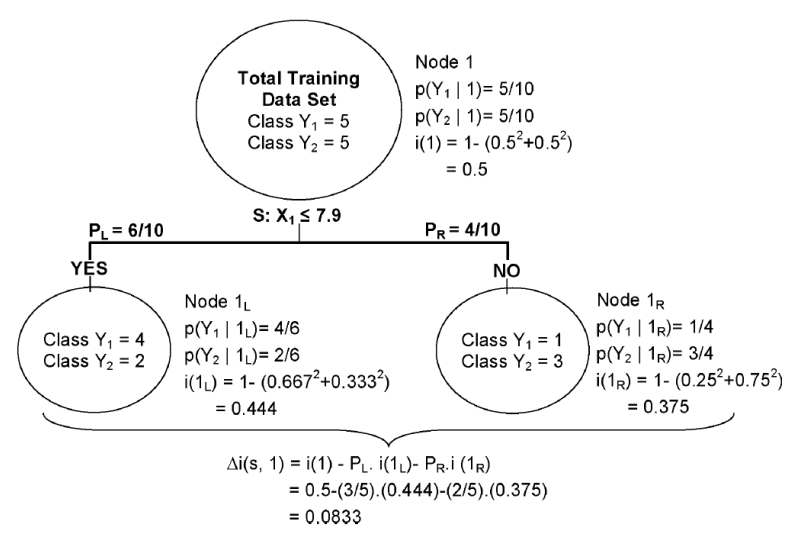

Figure 2. Example of a DT structure with 2 class problem

\section{Numerical Studies}

As proposed method using features that are sampled before main circuit breaker, a microgrid is introduced that has 4 sections included 2 DG and a battery supply with some loads that could be a likely micro-grid. The distribution network derived from system shown in Fig.3 is simulated. It is a system with four areas including a diesel generator, a wind turbine, a storage area (Ni-Cd battery) and some loads. Numerical studies are carried out using Matlab/Simulink software.

Microgrid could work itself, for example, Fig. 4 and 5 show changing in load and synchronous generator power when microgrid is isolated from grid. Loads are decreased, but power producing of synchronous generator increased because of charging battery.

The system has one switch SC1 (which is kept normally closed), making it possible to change the network configuration from in two classes of events, namely "non-islanding" and "islanding" were considered. The non-islanding cases simulated include: 1) normal operation; 2) temporary faults, including three phase to ground, three phase, line to line, and line to ground; 3) switching of loads; and 4) power decreasing of gird.

The data were collected at different loading conditions and under different system faults obtained by opening and closing switch SC1. A total of 200 islanding and non-islanding cases were simulated and the three-phase currents and voltages measured at the terminals of SC1 were recorded. Nearly $70 \%$ of the data, which includes 140 islanding and non-islanding cases, were used for training. The remaining cases were used as testing data in DT classification and $15 \%$ of the data were used for training, and $15 \%$ of the remaining data, were used 
for validating ANN classification. Testing data were extracted randomly from each category of events to ensure

testing against all types of transient events.

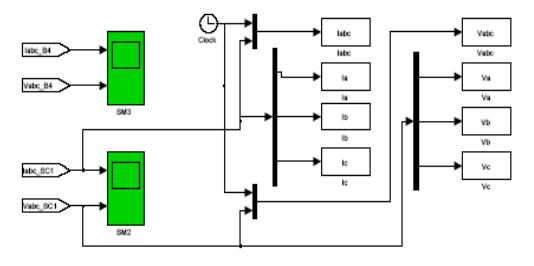

Islanding Microgrid Network Test
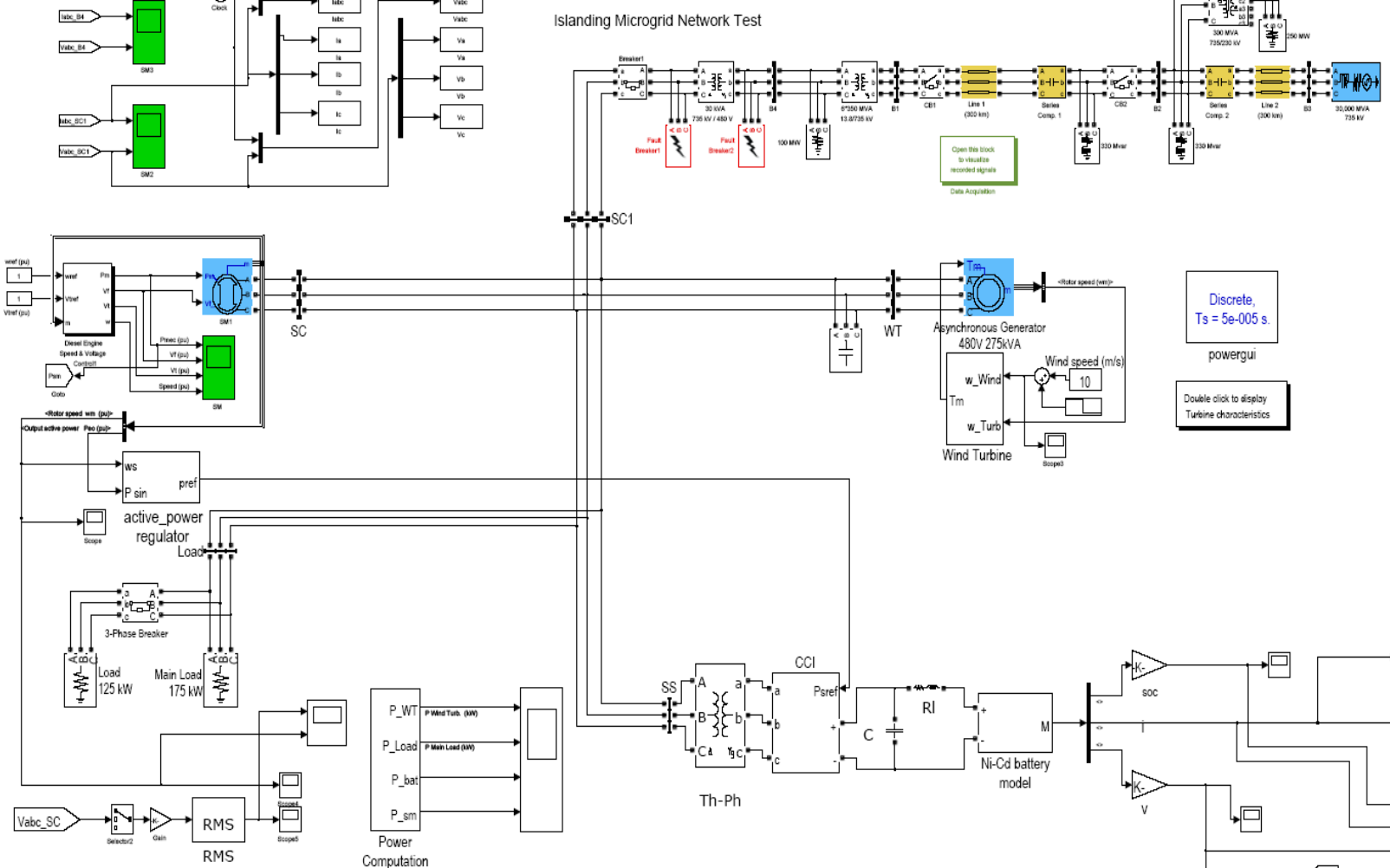

The DWT and ANN were used to extract the features for the classifiers. Phase current and voltage signals were sampled at $10 \mathrm{kHz}$ and every $0.05 \mathrm{sec}$ is used for calculation. The DWT was performed on the sampled waveforms with the Daubechie's 4 (Db4) mother wavelet. The approach for selecting the mother wavelet and sampling frequency was a trial-and-error procedure combined with prior experience. The successful application of $\mathrm{Db} 4$ for characterizing power system transients is reported in many studies [31]-[34].

The choice of $10-\mathrm{kHz}$ sampling frequency for detailed studies was based on the accuracy of classification, speed of detection, and hardware cost/capability required for real-time implementation. The original signals and the detail wavelet coefficients of the current and voltage measured in SC1 terminals for 1) an islanding event, 2) a line-to-ground with impedance fault, and 3) a load trip situation.

For example voltage and current of a three phase fault to ground with $0.01 \mathrm{Ohm}$ resistance is shown in Fig.6. Analysis of current with Db4 in level 4 is shown in Fig.7 and finally sum of discrete wavelet transform current and voltage Calculation energy can be defined respectively as:

$$
\begin{aligned}
& \text { EID4 }=\text { Energy_Ia_D4 }+ \text { Energy_Ib_D4 }+ \text { Energy_Ic_D4 } \\
& \text { EID3 }=\text { Energy_Ia_D3 }+ \text { Energy_Ib_D3 }+ \text { Energy_Ic_D3 } \\
& \text { EID2 }=\text { Energy_Ia_D2 }+ \text { Energy_Ib_D2 }+ \text { Energy_Ic_D2 } \\
& \text { EID } 1=\text { Energy_Ia_D1 }+ \text { Energy_Ib_D1 }+ \text { Energy_Ic_D1 } \\
& \text { EVD4 }=\text { Energy_Va_D4 + Energy_Vb_D4 + Energy_Vc_D4 } \\
& \text { EVD3 }=\text { Energy_Va_D3 }+ \text { Energy_Vb_D3 }+ \text { Energy_Vc_D3 } \\
& \text { EVD2 }=\text { Energy_Va_D2 }+ \text { Energy_Vb_D2 }+ \text { Energy_Vc_D2 } \\
& \text { EVD1 }=\text { Energy_Va_D1 }+ \text { Energy_Vb_D1 }+ \text { Energy_Vc_D1 }
\end{aligned}
$$

The disturbance is applied at $0.2 \mathrm{~s}$, and DWT coefficients are shown on an expanded scale that covers a time window of $0.05 \mathrm{~s}$, extending from $0.2 \mathrm{~s}$ to $0.25 \mathrm{~s}$. In addition to the obvious variations in the range of amplitudes, there are other noticeable differences between the islanding and non-islanding events. Furthermore, it is clear that different non-islanding events themselves hold identities. Thus, an approach, such as a simple threshold, cannot be used to distinguish between islanding and non-islanding events. Consequently, a more sophisticated method, involving pattern recognition, is required. 


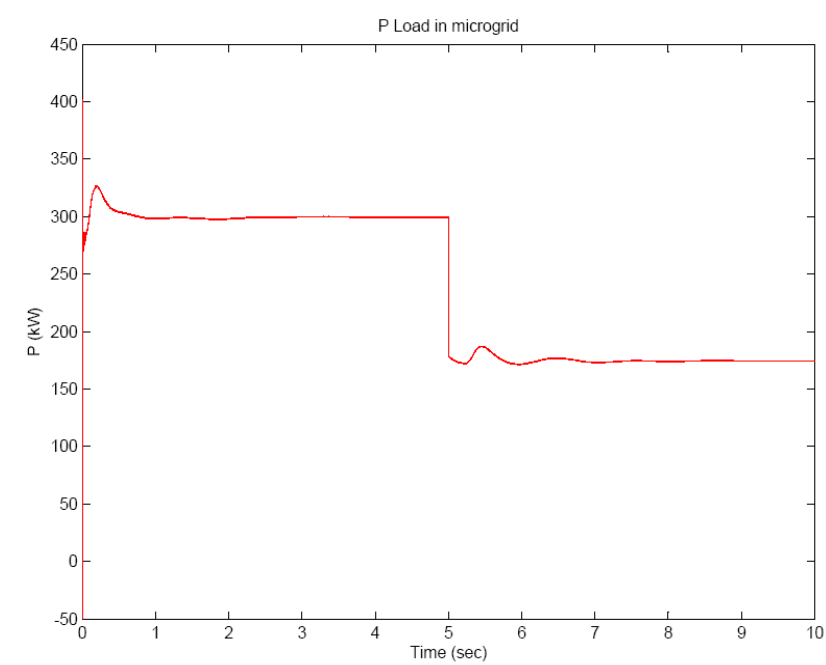

Figure 4. Amount of load is changed in microgrid after $5 \mathrm{sec}$.

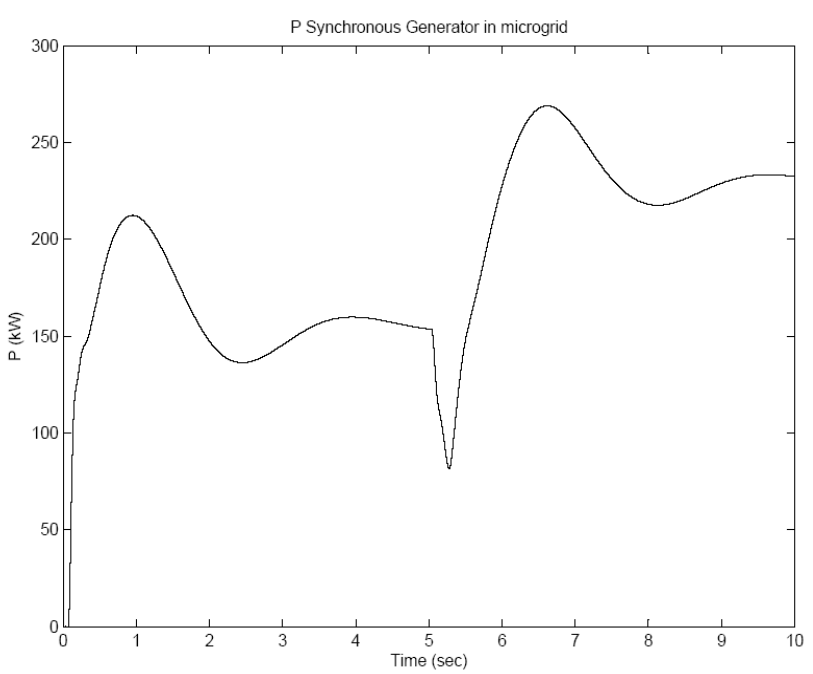

Figure 5. Power of synchronous generator varieties in microgrid after $5 \mathrm{sec}$.

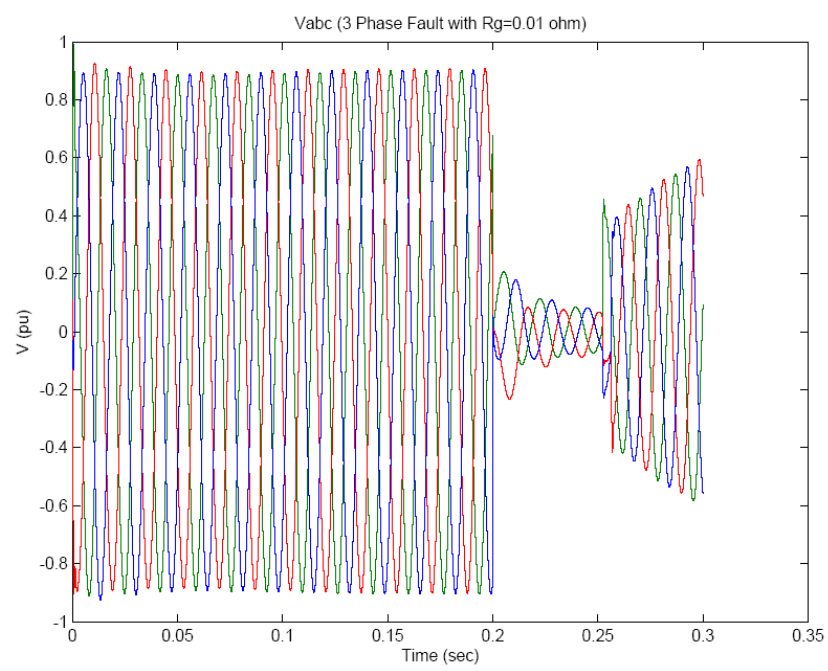

(a)

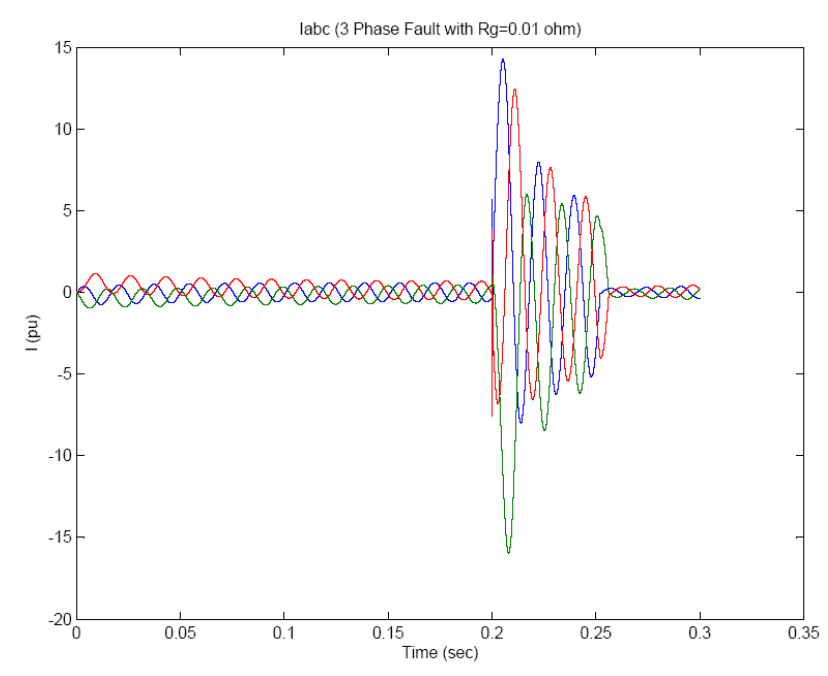

(b)

Figure 6. A three phase fault to ground with $0.01 \mathrm{Ohm}$ resistance (a) Voltage (b) Current.
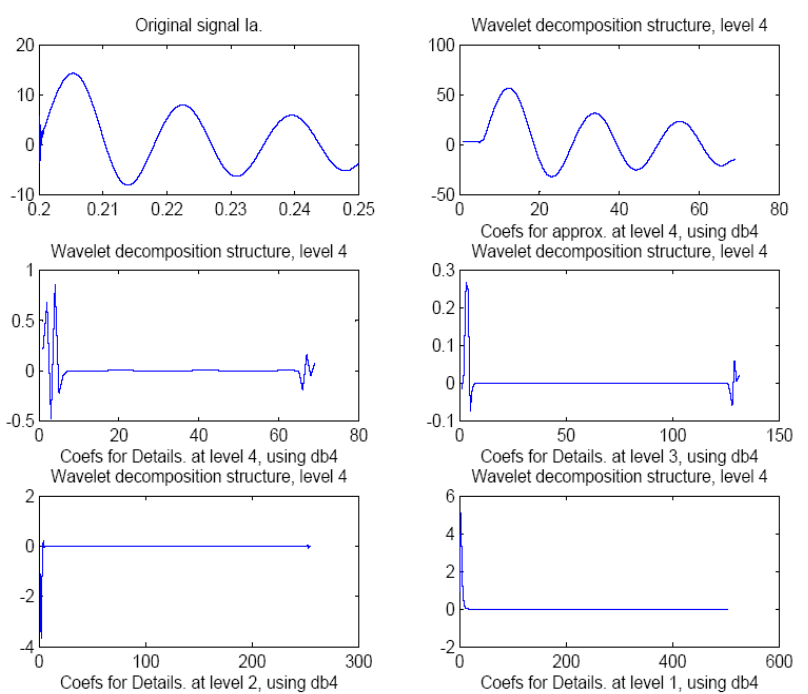

Figure 7. A three phase fault to ground with $0.01 \mathrm{Ohm}$ resistance (a) Voltage (b) Current.

Direct use of wavelet coefficients, which are essentially waveforms, as inputs to a classifier (in this case, to a DT) is impractical. Thus, energies associated with the wavelet coefficients in a time window that encompass the transient were used as features for the classifier. Wavelet energy is obtained by integrating the square of the wavelet coefficient over a time window of $0.05 \mathrm{~s}$. The analysis uses a moving window, thus preserving the temporal information. This time window length was selected after preliminary investigations as a compromise between the accuracy and response time. At each decomposition level, the energies of the three phases were added to form a combined "three-phase energy" value in the particular frequency band. This feature extraction method is illustrated in Fig. 8. Only the decomposition of Phase-a current is shown in detail to reduce the complexity of the figure. The so-calculated "three-phase energy" values of the currents and voltages create a $12-\mathrm{D}$ feature space (6 
levels of currents 6 levels of voltages) for each generator, if the output of six levels from the DWT is used.

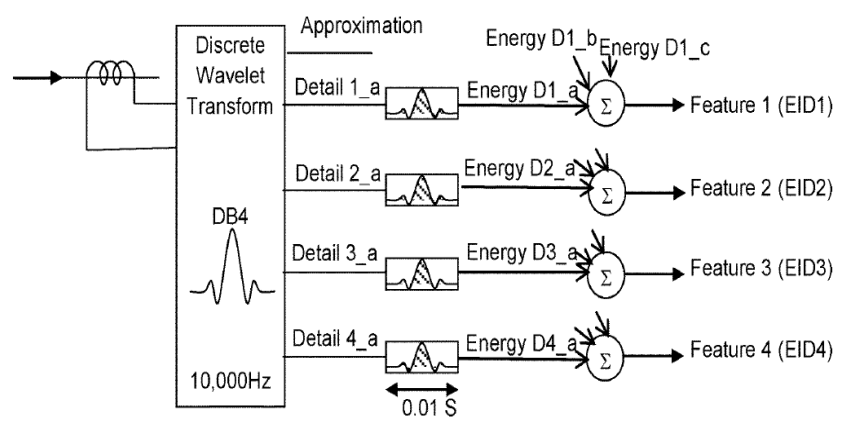

Figure 8. Feature extraction methodology [20].

Initially, a DT and an ANN were trained for microgrid using all 8 features that are calculated from (4) and (5). In Table I, EVD and EID denote the energy values of the level wavelet coefficient of the voltages and the currents, respectively for 4 examples. An analysis of Table I shows that the relevancy of some of the features is very low and they can be omitted without significantly reducing the accuracy.

Table 1. EVD and EID values of the level wavelet coefficient of the voltages and the currents.

\begin{tabular}{lllll}
\hline & Ex.1 & Ex.2 & Ex.3 & Ex.4 \\
\hline EID4 & 0.012403 & 0.012433 & 1.88537 & 3.976009 \\
EID3 & 0.007196 & 0.007209 & 1.870209 & 0.600069 \\
EID2 & 0.000735 & 0.000737 & 11.76482 & 44.46214 \\
EID1 & $2.29 \mathrm{E}-06$ & $2.30 \mathrm{E}-06$ & 9.74209 & 1857.722 \\
EVD4 & 0.029793 & 0.029556 & 0.383118 & 0.446404 \\
EVD3 & 0.017385 & 0.017396 & 2.535627 & 3.041684 \\
EVD3 & 0.001778 & 0.001784 & 0.451739 & 0.833201 \\
EVD3 & $6.02 \mathrm{E}-06$ & $6.05 \mathrm{E}-06$ & 0.040049 & 0.310759 \\
State & 0 & 0 & 1 & 1 \\
\hline
\end{tabular}

\subsection{Classification with Artificial Neural Networks}

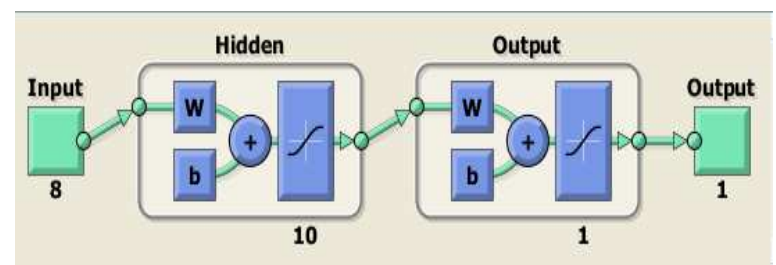

Figure 9. Structure of $A N N$.

Artificial neural network that is used for classification is a feed-forward network with 8 inputs and 1 output. It has 2 layers and training that is used for this network is a Levenberg-Marquardt using Mean Square Error (mse) for decreasing of errors. Structure of ANN is shown in Fig.9 and error histogram is shown in Fig.10. Under these structure and results, accuracy of this ANN is about $84 \%$.

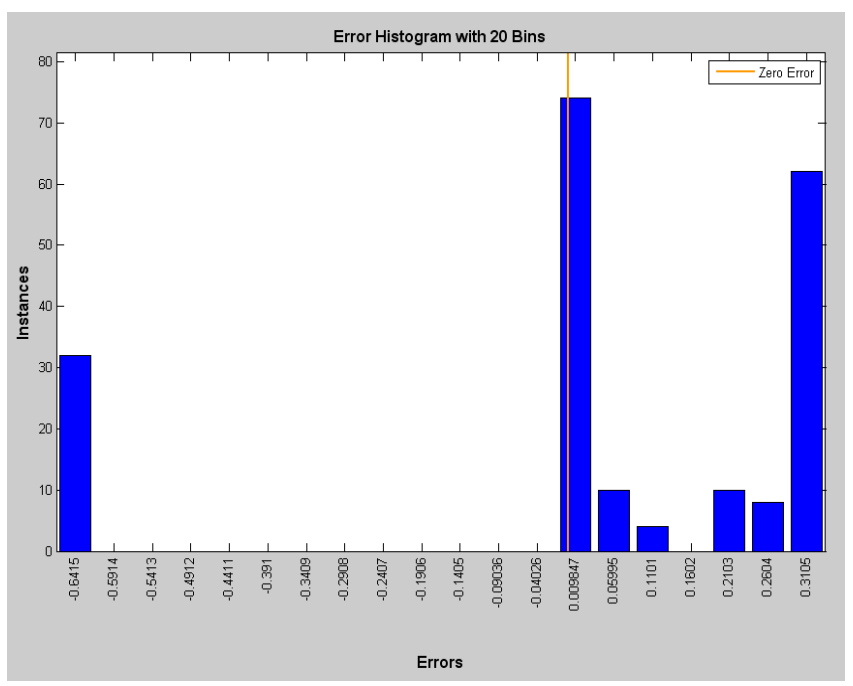

Fig 10. Error histogram of $A N N$.

\subsection{Classification with Decision-Tree}

The DT classifier trained for all states. With the testing data for the microgrid, it achieved an overall accuracy over $99 \%$ in identifying islanding events. Table II shows decision tree rules for islanding detection and Fig. 11 shows branch of decision tree.

Table 2. Decision tree rules for islanding detection.

\begin{tabular}{|c|c|}
\hline & Decision tree for regression \\
\hline 1 & $\begin{array}{l}\text { if } x 6<0.0173962 \text { then node } 2 \text { elseif } x 6>=0.0173962 \text { then node } 3 \text { else } \\
0.84\end{array}$ \\
\hline 2 & $\begin{array}{l}\text { if } x 4<2.28792 \mathrm{e}-006 \text { then node } 4 \text { elseif } x 4>=2.28792 \mathrm{e}-006 \text { then node } \\
5 \text { else } 0.545455\end{array}$ \\
\hline 3 & $\begin{array}{l}\text { if } x 6<0.0174052 \text { then node } 6 \text { elseif } x 6>=0.0174052 \text { then node } 7 \text { else } \\
0.985075\end{array}$ \\
\hline 4 & fit $=1$ \\
\hline 5 & $\begin{array}{l}\text { if } x 7<0.00178427 \text { then node } 8 \text { elseif } x 7>=0.00178427 \text { then node } 9 \\
\text { else } 0.387755\end{array}$ \\
\hline 6 & $\begin{array}{l}\text { if } x 1<0.0124798 \text { then node } 10 \text { elseif } x 1>=0.0124798 \text { then node } 11 \\
\text { else } 0.888889\end{array}$ \\
\hline 7 & fit $=1$ \\
\hline 8 & fit $=0$ \\
\hline 9 & $\begin{array}{l}\text { if } x 5<0.0295559 \text { then node } 12 \text { elseif } x 5>=0.0295559 \text { then node } 13 \\
\text { else } 0.76\end{array}$ \\
\hline 10 & fit $=1$ \\
\hline 11 & fit $=0$ \\
\hline 12 & fit $=1$ \\
\hline 13 & $\begin{array}{l}\text { if } x 5<0.0295561 \text { then node } 14 \text { elseif } x 5>=0.0295561 \text { then node } 15 \\
\text { else } 0.5\end{array}$ \\
\hline 14 & fit $=0$ \\
\hline 15 & fit $=1$ \\
\hline
\end{tabular}




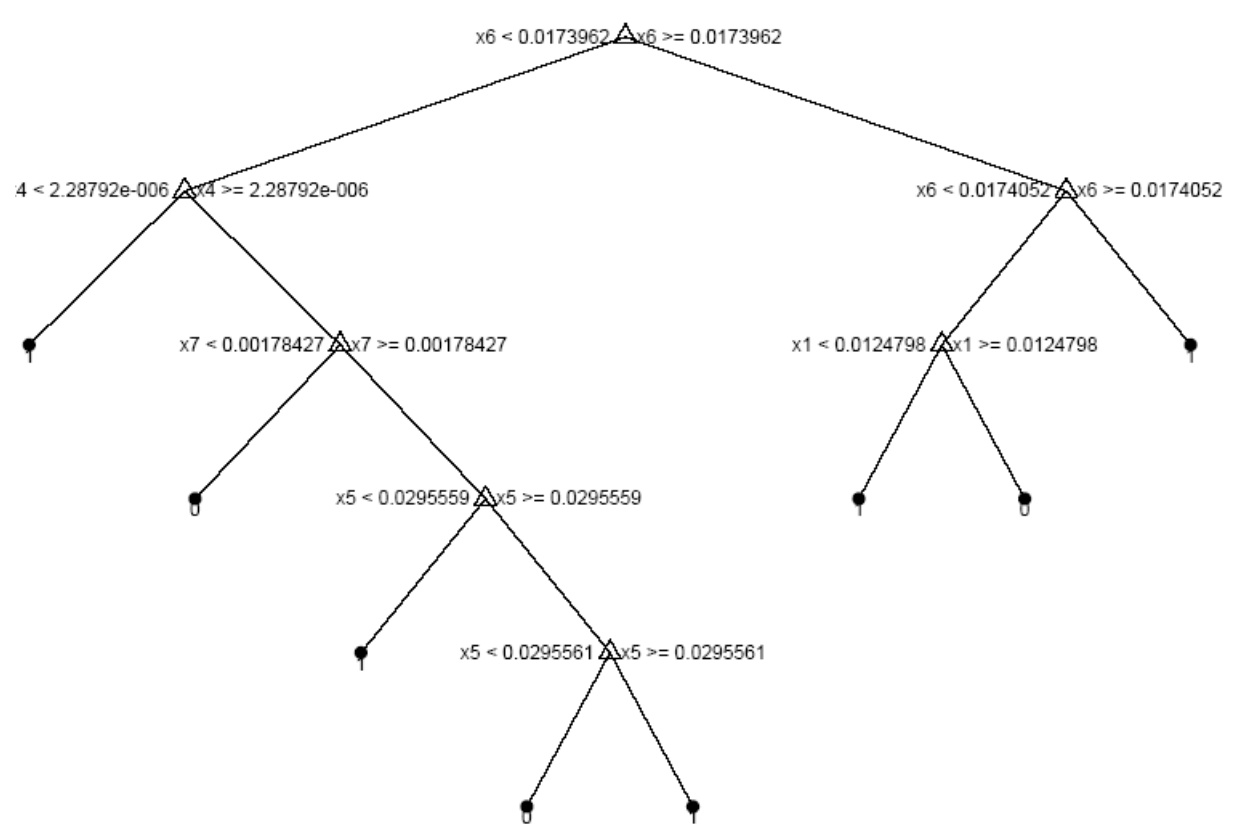

Figure 11. Decision-tree

\section{Conclusion}

This paper dealt with islanding detection based on discrete wavelet transform. Changing the location of current and voltage signals measuring point after the utility circuit breaker makes this method more effective for islanding detection in the network with multiple DGs. Islanding detection time is decreased by using the DWT for feature extraction.

A trained DT classifier is able to successfully categorize the transient generating events as "islanding" or "non-islanding" using the energy associated with the wavelet coefficients. When tested with a large number of test cases, the proposed technique shows more than $99 \%$ overall classification accuracy beside ANN that only shows $84 \%$ classification accuracy.

As it mentioned, for detecting islanding, this paper presented an online measurement method based on wavelet transform for current and voltage analysis and results could be described in brief:

1. Islanding detection time is decreases using signals before main circuit breaker.

2. Reliability is increased using transient signals that are sent from network with less noise effects besides using further noisy signals in microgrid in other methods.

Several issues remain for the future works within distribution networks and microgrids, such as other classification methods, using fuzzy method in DT classification, using other sample signals such as frequency deviation, and using other time frequency analysis such as S-transform, Hyperbolic S-transform, and TT-Transform instead of wavelet transform. These are the subjects of ongoing research.

\section{References}

[1] UL 1741, "Inverters, converters, and controllers for use in independent power systems," 2002.

[2] IEEE, Std. 1547, "IEEE standard for interconnecting distributed resources with electric power systems," 2003.

[3] F. Wang and Z. Mi, "Passive islanding detection method for grid connected PV system," Proc. Int. Conf. on Industrial and Information Systems, pp. 409-412, June 2009.

[4] M. E. Ropp, M. Begovic, and A. Rohatgi, "Analysis and performance assessment of the active frequency drift method of islanding prevention," IEEE Trans. Energy Convers., vol. 14, no. 3, pp. 810-816, September 1999.

[5] W. Bower and M. Ropp, "Evaluation of islanding detection methods for photovoltaic utility-interactive power systems," Int. Energy Agency,Tech. Rep. IEA PVPS T5-09, March 2002.

[6] Europe 2020, "A strategy for smart sustainable and inclusive growth", available at: http://ec.europa.eu/eu2020/index_en.htm.

[7] “Technology Action Plan: Smart Grids". Report to the Major Economies Forum on Energy and Climate, December 2009.

[8] Interconnecting Distributed Resources With Electric Power Systems, IEEE Std. 1547-2003, 2003.

[9] R. A. Walling and N. W. Miller, "Distributed generation islanding - Implications on power system dynamic performance," in Proc. IEEE Power Eng. Soc. Summer Meeting, 2002, vol. 1, pp. 92-96.

[10] W. Freitas, W. Xu, C. M. Affonso, and Z. Haung, "Comparative analysis between ROCOF and vector surge relays for distributed generation applications," IEEE Trans. Power Del,, vol. 20, no. 2, pt. 2, pp. 1315-1324, Apr. 2005. 
[11] J. E. Kim and J. S. Hwang, "Islanding detection method of distributed generation units connected to power distribution system," in Proc. PowerCon, Perth, Western Australia, 2000, vol. 2, pp. 643-647.

[12] W. Freitas, Z. Huang, and W. Xu, "A practical method for assessing the effectiveness of vector surge relays for distributed generation applications," IEEE Trans. Power Del., vol. 20, no. 1, pp. 57-63, Jan. 2005.

[13] T. Funabashi, K. Koyanagi, and R. Yokoyama, "A review of islanding detection methods for distributed resources," in Proc. IEEE Bologna Power Tech Conf. , 2003, vol. 2, pp. 6-11.

[14] H. H. Zeineldin, E. F. El-Saadany, and M. M. A. Salama, "Distributed generation micro-grid operation: Control \& protection," in Proc. Power Systems Conf:: Advanced Metering, Protection, Control, Communication, and Distributed Resources, 2006, pp. 105-111.

[15] Y.-H. Liy, T.-S. Luor, S.-J. Huang, and J.-M. Lin, "Method and System for Detecting Stand-Alone Operation of a Distributed Generating System,” U.S. Patent 7342 758, Mar. 2008.

[16] C.-T. Hsieh, J.-M. Lin, and S.-J. Huang, "Enhancement of islanding- detection of distributed generation systems via wavelet transform-based approaches," Int. J. Elect. Power Energy Syst., vol. 30, no. 10, pp. 575-580, Dec. 2008.

[17] Pigazo, V. M. Moreno, M. Liserre, and A. Dell'Aquila, "Waveletbased islanding detection algorithm for single-phase PV distributed generation systems," in Proc. IEEE Int. Symp. Industrial Electronics, Vigo, Spain, pp. 2409-2413.

[18] K. El-Arroudi, G. Joós, I. Kamwa, and D. T. McGillis, "Intelligentbased approach to islanding detection in distributed generation," IEEE Trans. Power Del., vol. 22, no. 2, pp. 828-835, Apr. 2007.

[19] Arachchige, L.W. ; Rajapakse, A. "A pattern recognition approach for detecting power islands using transient signals Part I: Design and implementation" Power and Energy Society General Meeting, 2011 IEEE , pp. 1, 24-29 July 2011.

[20] Vatani, M.R. ; Sanjari, M.J. ; Gharehpetian, G.B. ; Moghani, J.S. "A new fast approach for islanding detection in the distribution networks with high penetration of distributed generation" Smart Grid Conference (SGC), 2013, pp. 241 245, 17-18 Dec. 2013.

[21] Gaouda A M, Salama M M A, Sultan M R, et al. "Application of multi resolution signal decomposition for monitoring short-duration variations in distribution systems," IEEE Trans. on Power Delivery, vol.15, no.2, pp. 478-485, 2000 .

[22] E. F. El-Saadany, T. K. Abdel-Galil and M. M. A. Salama, "Application of Wavelet transform for assessing power quality in medium voltage industrial distribution system," in Proc. of 2001 IEEE/PES Transmission and Distribution Conference and Exposition, vol. 1, pp. 427-432, 28 Oct.-2 Nov. 2001.

[23] Y. Yohannes and P. Webb, "Classification and regression trees, cart TM-A user manual for identifying indicators of vulnerability to famine and chronic food insecurity," Microcomputers in Policy Research 3, Int. Food Policy Res. Inst., 1999.

[24] M. Tarafdar Haque, and A.M. Kashtiban," Application of Neural Networks in Power Systems; A Review" World Academy of Science, Engineering and Technology 6, 2005.

[25] Hagan, M.T., H.B. Demuth, and M.H. Beale, Neural Network Design, Boston, MA: PWS Publishing, 1996.

[26] Y. Sheng and S. M. Rovnyak, "Decision tree-based methodology for high impedance fault detection," IEEE Trans. Power Del., vol. 19, no. 2, pp. 533-536, Apr. 2004.

[27] Leo, J. Friedman, R. Olshen, and C. Stone, Classification and Regression Trees. Belmont, CA: Wadsworth, 1984.

[28] Steinberg and P. Colla, CART: Tree-Structured Non-Parametric Data Analysis. San Diego, CA: Salford Systems, 1995.

[29] Y. Yohannes and P. Webb, "Classification and regression trees, cart TM-A user manual for identifying indicators of vulnerability to famine and chronic food insecurity," Microcomputers in Policy Research 3, Int. Food Policy Res. Inst., 1999.

[30] L. Bel, D. Allard, J. M. Laurent, R. Cheddadi, and A. Bar-Hend, "CART algorithm for spatial data: Application to environmental and ecological data," Comput. Stat. Data Anal., vol. 53, no. 8, pp. 3082-3093, Jun. 2009.

[31] H. Kim and R. Agganrval, "Wavelet transforms in power systems - Part 1: General introduction to the wavelet transforms," IEEE Power Eng. J., vol. 14, no. 2, pp. 81-87, Apr. 2000 .

[32] C. H. Kim and R. Agganrval, "Wavelet transforms in power systems - Part 2: Examples of application to actual power system transients," IEEE Power Eng. J., vol. 15, no. 4, pp. 193-202, Apr. 2000.

[33] H. A. Darwish, M. H. Farouk, A.-M. I. Taalab, and N. M. Mansour, "Investigation of real-time implementation of DSP-based dwt for power system protection," in Proc. IEEE Power Eng. Soc. Transmission and Distribution Conf. Exhibit. ,pp. 1258-1263, 2005/2006.

[34] N. Perera and A. D. Rajapakse, "Rapid isolation of faults in power networks with distributed generators,"M.Sc. dissertation, Univ. Manitoba, Winnipeg, MB, Canada, May 2007. 